Safdar, K., A. Emrouznejad, Dey, P. (2020) An Optimized Queue Management System to

\title{
An Optimized Queue Management System to Improve Patient Flow in the Absence of Appointment System
}

\author{
K.A. Safdar, A. Emrouznejad, P.K. Dey \\ Aston Business School, Aston University, UK
}

\begin{abstract}
Purpose: The aim of this research study is to develop a queue assessment model to evaluate the inflow of walk-in outpatients in a busy public hospital of an emerging economy, Pakistan, in the absence of appointment systems; as well as, constructing a dynamic framework dedicated towards the practical implementation of the proposed model, for continuous monitoring of the queue system.
\end{abstract}

Methodology: The current study utilizes Data Envelopment Analysis (DEA) to develop a combined Queuing-DEA model as applied to evaluate the wait times of patients, within different stages of the Outpatients' department at the Combined Military Hospital (CMH) in Lahore, Pakistan; over a period of 7 weeks (23 $3^{\text {rd }}$ April to $28^{\text {th }}$ May 2014). The number of doctors/personnel and consultation time were considered as outputs, where consultation time was the non-discretionary output. The two inputs were wait time and length of queue. Additionally, VBA programming in Excel has been utilized to develop the dynamic framework for continuous queue monitoring.

Findings: The inadequate availability of personnel (doctors/pharmacists) was observed as the critical issue for long wait times, along with overcrowding and variable arrival pattern of walkin patients. The DEA model displayed the 'required' number of personnel, corresponding to different wait times, indicating queue build-up.

Originality/Value: The current study develops a queue evaluation model for a busy outpatients' department in a public hospital, where all patients are walk-in and no appointment systems. This model provides vital information in the form of 'required' number of personnel which allows the administrators to control the queue pre-emptively minimizing wait times, with dynamic staff allocation. Additionally, the dynamic framework specifically targets practical implementation in resource-poor public hospitals of emerging economies for continuous monitoring of queues.

Keywords: Public health system, Waiting time, Data Envelopment Analysis, Queuing theory, Absence of appointment system, Developing Economy

Classification: Case Study 


\section{Introduction}

Public hospitals in emerging economies are unable to cater to the needs of majority of population, due to lack of infrastructure, limited funds, shortage and inadequate allocation of resources, lack of planning and organisation creating an imbalance between distribution of resources (Berenguer .,., 2016; Chandra 2015; Driouchi 2014) and demand of high quality services by patients (Susanti et al., 2020; Ahmed et al., 2017). Emerging economies face additional issues including high level of population and growth rate, low literacy rate, high rate of diseases, lack of medical equipment and aftercare facilities, late arrival of doctors, disparity in access to services and social/cultural barriers; resulting in increased patient suffering and inconvenience (Mensah et al., 2015; Bhattacharjee and Ray 2014). One such emerging economy is Pakistan, which is the sixth most highly populated country in the world, with a population of nearly 220.1 million (Qureshi 2020). Nearly $60 \%$ of the population resides in rural areas (World Bank data 2018). The literacy rate in Pakistan is only around 60\% (PSLM Survey 2018-19); which is much lower than almost 100\% literacy rate in developed nations, and even lower than some other emerging economies. Pakistan faces an increasing challenge of improving health outcomes due to a high population rate and increasing burden of diseases leading to patient dissatisfaction (Javed and Ilyas 2018; Jalal and Haq, 2014) and poor infrastructure with number of beds and physicians are 6 and 9 (per 10000 of population) on average respectively; which is lower than many developed countries (WHO Statistics 2015 and 2019). The current study addresses the issue of queuing problem from an emerging economy perspective, in a busy hospital of Pakistan.

Low patient wait time is one of the significant criteria which not only assesses patient satisfaction (Oche and Adamu 2014) but also service quality and efficiency, and capacity planning (Mensah et al., 2015; Kuiper et al., 2019). One of the major characteristics prevailing within all large public hospitals in emerging economies such as Pakistan, is the absence of Appointment systems in Outpatients' department; creating hurdles in the efficient management of patients resulting in excessive queuing (Wei et al., 2015; Safdar et al., 2016). Although the queuing problem has been widely researched in healthcare, however, only a limited number have considered walk-in patients, and even these have emphasized on improving an existing Appointment system considering walk-ins. The study by Bailey (1952) was one of the first to evaluate an appointment system. This study along with Fetter and Thompson (1966) and Klassen and Rohleder (1996) recommended improved appointment system mainly by examining the relationship between average wait times of patients and doctors' idle time. Zhu et al., (2012) concluded that uneven distribution of appointment slots, late start of sessions and unused sessions; lead to long wait time. Barghash and Saleet (2018) evaluated the impact of high lateness and no show percentages when setting appointment schedules. Nappo and Ross (2020) propose staggered appointment schedules with small group of patients rather than two big shifts to increase efficiency and minimize wait times. Klassen and Yoogalingam (2019) investigated the effect of adding mid-level service providers (assistants and nurse practitioners) on patient wait time, flow time and physician service time. Cayirli and Veral (2003), Lakshmi and Iyer (2013) and Marynissen and Demeulemeester (2019) provide a comprehensive survey on appointment scheduling problems in an Outpatient setting. But in emerging economies such 
as Pakistan, all outpatients are walk-in with variable arrival pattern, hence a dynamic framework which continuously monitors the patient flow system will be more effective.

A limited number of studies have been carried out in emerging economies, which specifically considered walk-in patients within the outpatients' department of large public hospitals. Huarng and Lee (1996) and Aharonson-Daniel et al., (1996), briefly discussed the option of implementing complete appointment systems to minimize wait times, in their studies in Taiwan and Hong Kong respectively. In studies in Nigeria and Ghana respectively, Oche and Adamu (2014) and Mensah et al., (2015) emphasized on training and supervision of health workers for use of electronic based systems to implement triage system, improve service and reduce wait times. In a study in the Sri Lankan National Hospital Outpatients' department, Dilrukshi et al., (2016) suggested that more doctors can be assigned to the roster to reduce wait times. Aeenparast et al., (2019) concluded that physician delay was a major factor in increased outpatient wait times in a General hospital in Iran. In a recent study in Pakistan, Kalwar et al., (2020) evaluated the wait times within a Gastronlogy department for patients with appointments suggesting that doctors should sit in for longer hours. Although these suggestions are reasonable, but not currently implementable as almost all public hospitals of developing economies are highly unorganised, overcrowded, and all patients are walk-in with variable arrival pattern. This necessitates the formulation of a flexible model which continuously evaluates the current walk-in patient flow system, which is the objective of the current study.

The existing studies are dedicated towards evaluating an existing appointment system which incorporates scheduled and walk-in patients. In emerging economies, only a few studies have evaluated wait times of walk-in patients and provided recommendations; however, there is a need to develop a framework which continuously monitors the queue in overcrowded public hospitals with variable arrival patterns. but they are very less in number. The current study addresses this significant issue by developing a queue assessment model for a busy public hospital of an emerging economy, Pakistan, in the absence of appointment systems; and proposes a framework which can continuously act to control long queues more effectively.

\section{Methodology}

A number of different modelling techniques have been used by researchers to evaluate existing queue systems, where Discrete-Event Simulation (Harper and Gamlin 2003; Anderson et al., 2015; Viana et al., 2020; Perez et al., 2020) and Queuing Models (Brahimi and Worthington 1991; Peter and Sivasamy 2019; Jiang et al., 2019) are among the most commonly utilized, as well as Predictive models (Kortbeek et al., 2015) and Optimization models (Wang et al., 2020; Lou et al., 2020). The current study employs the specialized efficiency technique of DEA to develop a model for queue assessment presenting a novel application of this modelling tool, and providing evidence of its effectiveness with regard to queue management.

\subsection{Queuing-DEA Model Development}


Over the years, different Operational Research (OR) modelling techniques have been used to assess the queue problem. However, many of these techniques utilize 'average' values and 'pre-specified' statistical distributions to represent a queue system, as demonstrated by previous works. Anderson et al., (2015) utilized the mean service time, and employed Uniform distribution to estimate this variable. Mital (2010) assumed that service times as well as interarrival times follow Exponential distribution. Mandelbaum et al., (2012) used Poisson and Exponential distributions for arrival pattern of patients and length of stay respectively. Feldman et al., (2014) used Poisson distribution for the number of appointment requests by patients in a day. For large public hospitals in emerging economies, such as Pakistan, the arrival pattern of patients is highly unpredictable due to absence of appointments. Therefore, an average value of queuing variables and pre-specified statistical distributions might not accurately represent the queue system. It lacks the detail required to analyse the disorganized queue problem of this magnitude. Hence, for the current study, the efficiency assessment technique of Data Envelopment Analysis (DEA) has been employed which has the capability to address these issues, making it suitable to evaluate a complex queue system.

Data Envelopment Analysis is a multiple criteria decision-making technique, which is used to model the relationship between multiple inputs and outputs in order to determine the efficiency level of all units, known as decision-making units (DMUs) (Hollingsworth 2012). It is a nonparametric technique which does not require any a priori specification of the functional form or distribution of data, efficiency frontier and efficiency scores. Additionally, DEA analyses 'each' unit separately rather than providing an average across all data points (Charnes et al., 1994). A DEA model identifies benchmarks for improvements for the inefficient units. Additionally, the most significant advantage of this modelling technique is that it provides a 'target' value for each input and output for each DMU, showing the exact amount required for the unit to become fully efficient. This additional information can assist policy-makers in decision-making, by highlighting the bottlenecks and provides a guideline for improving efficiency (Liu et al., 2013a; Lee and Kim 2014). Therefore, these characteristics render DEA analysis as highly appropriate for problems such as a complex queue system where data is extremely variable, and lacks a proper strategy and guidance (see Safdar et al., (2016) for details on the traditional model).

The development of the DEA model for queue assessment consists of different stages (as shown in Figure 1). The initial structure of the DEA model was developed first and validated (as shown by Safdar et al., 2016), which guided the data collection process. Based on the preliminary results obtained from the data gathered, a refined DEA model was constructed, which is then used to evaluate the queue system at the designated hospital in Pakistan.

*Insert Figure 1 here

\subsection{Preliminary Findings and Queuing-DEA Model Inputs/Outputs}


Preliminary observations demonstrated that there are a number of different pathways that the outpatients are required to follow, from entry till exit in the patient flow system. When a patient first arrives at the hospital, he/she needs to be first examined by a General Duty Medical Officer (GDMO) (GP-equivalent doctor), and then directed accordingly, as shown in Figure 2.

\section{*Insert Figure 2 here}

A number of interesting findings are highlighted which along with real-time queuing data, are utilized to refine the DEA model to better suit the purpose of the current study. The inputs and outputs of the existing model are highlighted below:

\subsubsection{Output 1: Number of personnel (doctors/pharmacists)}

The most significant issue highlighted by observing the queue system, is the inadequate allocation of personnel. For instance, it was observed that the maximum load of outpatients is during early morning (7-8:30 am) at GDMO clinic and the busiest Specialist OPD, with at least 50 patients waiting at one time. However, only 2 to 3 doctors were working when the session starts at 08:30am. As the availability varied over the day, it was observed that at around 10:30$11 \mathrm{am}$, the availability increases up to five in the Specialist OPD. But, by this time, a long queue of patients has already built up with most patients waiting since early morning. The lack of sync as observed between the availability of staff and excessive queuing, which is further worsened by the lack of appointments and irregular arrival behaviour of patients, is a critical issue which needs to be addressed. In an ideal situation, more doctors can be employed and number of clinics enhanced. But with limited staff, along with long queues, it is essential to determine the exact 'required' availability of doctors/personnel at a certain time, using certain criteria. In this case, the objective is to 'increase' the availability of personnel, hence, this variable has been added as an output. This is contradictory to previous queue management and DEA studies, where the aim is to 'minimize' the number of staff to improve efficiency. The administrators require a 'flexible' framework which is responsive to the rapidly varying wait times and load of patients, and provides a guideline to 'optimally' utilize personnel to minimize the queue.

\subsubsection{Output 2: Consultation time (Non-discretionary Output)}

Preliminary observations indicated that the consultation time was quite low, only 5-6 minutes on average, with the GDMO and even with the Specialist. One of the reasons for low consultation as observed is that most of the patients require a regular/follow-up check-up or simply a renewal of medicine prescriptions, which takes less time. Only in a few instances, first-time visits or patient requiring a detailed examination (children or aged people) took a bit longer (8-9 minutes) as observed; whereas patients requiring immediate care are transferred to the Emergency department instantly. Therefore, it is perhaps in the best interest not to actively influence further reduction in doctor-patient interaction time. Hence this variable has been included in the Queuing-DEA model as a 'non-discretionary' output with a view of keeping it constant; as opposed to previous DEA studies where 'non-discretionary' inputs are considered 
such as treatment population (Mitropoulou et al., 2013; Bastian et al., 2017) and number of beds (Herwartz and Schrumann 2014).

\subsubsection{Input 1: Wait time}

The initial findings indicated that the Radiology/Pathology department (Wait 3 as shown in Figure 1) is quite streamlined, with comparatively low wait times (an hour on average) and little variation. The main reasons in this regard are probably a limited number of patients requiring tests/investigations or since all OPDs do not have sessions on the same day, therefore, the load of patients is distributed among different days of the week. Additionally, low wait times were observed at the reception desk of each department, and same-day second consultations with GDMOs/Specialists (as they are given priority). However, high wait times were observed at the GDMO clinic, and some Pharmacy counters and Specialist OPDs (Wait 1, Wait 2 and Wait 4 respectively as shown in Figure 1). Therefore, for the purpose of this study, the wait time of Specialist OPD will be evaluated with wait time added as an input (same process for GDMO clinic and Pharmacy). As compared to previous studies, where the wait time was defined with regard to the appointment time, the current study considered the wait time of walk-in patients as the difference between the time a patient arrives and when he/she leaves. The inclusion of wait times will identify bottlenecks such that appropriate measures can be taken to improve the existing queue situation.

\subsubsection{Input 2: Length of Queue}

The initial observations showed that due to absence of appointment systems, the arrival pattern of patients is unpredictable; where the overload situation changes drastically in busy departments and a massive queue builds up just within few minutes. For instance, within the busiest Specialist OPD, it was observed that on busy days, the load of patients is extremely high with at least 50-60 patients waiting during early morning arriving between 7-7.30am. These patients are not examined before 10am. However, Pharmacy has less patients in the morning (only those patients who arrived just to collect medicines, either because they were unavailable on the day of consultation or they did not have time), but a drastic increase was observed at around 10am (normally Pharmacy is the last stage that patients visit). During busy days (usually Mondays and Fridays), it was observed that there were still about 40 patients waiting at around $12: 30 \mathrm{pm}$, and at this rate, the $40^{\text {th }}$ patient was served at around $3 \mathrm{pm}$.

Although the influx of walk-in patients cannot be controlled, however, there is a crucial need to 'measure' the level of overload of patients to assist in deriving appropriate strategies. Hence, the 'length of queue' (total number of patients waiting) has been included in the DEA model for each department as an 'indicator' of overcrowding.

\subsection{Mathematical Representation of Queuing-DEA Model}

Over the years, a number of DEA models with non-discretionary factors have been developed by researchers (Banker and Morey 1986; Golany and Roll 1993; Yang and Paradi 2003), 
however, Banker and Morey (1986) is the most frequently used model in literature (Muniz et al., 2006). The main benefit of Banker and Morey (1986) model is its computational advantages and its ability to include non-discretionary and discretionary variables simultaneously in the same DEA model, but either an input or output which is suitable for the current study (as consultation time is the only non-discretionary output).

The Queuing-DEA model developed for the current study is the output-oriented model with a non-discretionary output; where the objective is to 'maximize' the output (number of personnel) given the inputs (wait time and length of queue), with non-discretionary output (consultation time) unchanged. For this model, the constraint for the non-discretionary output is separate and does not consist of the efficiency variable $\phi$, as this variable is 'fixed' and will not affect efficiency. Additionally, the slack values of non-discretionary output will not be included in the objective function. Furthermore, the target value for the non-discretionary output will not include the efficiency variable $\phi$ and hence, will remain unchanged (Banker and Morey 1986; Cooper et al., 2011).

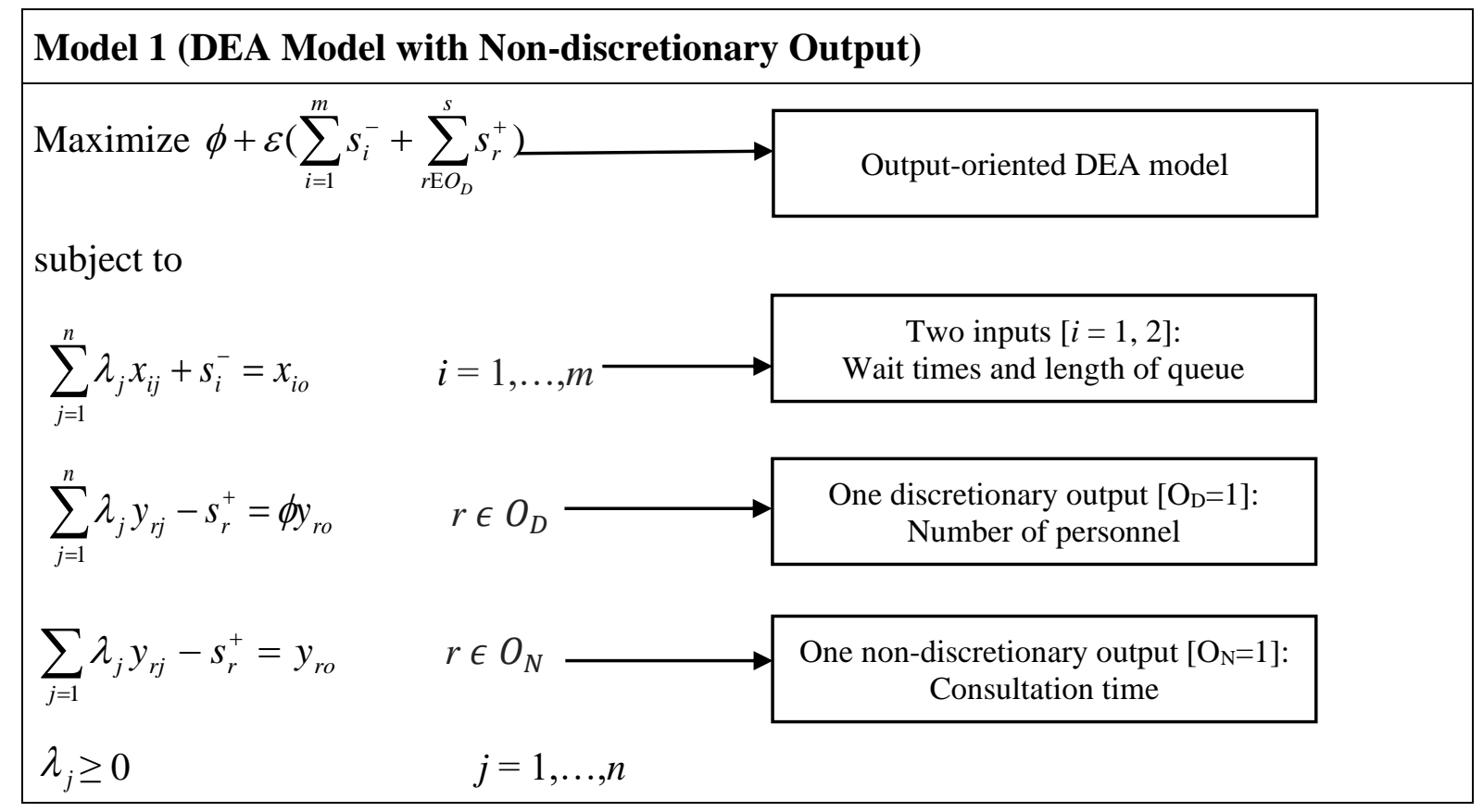

The Queuing-DEA model has been specifically developed to reflect the complex patient flow system at the busy public hospital. The most significant finding was the inadequate scheduling of doctors. Also, the consultation/service time was found to be extremely low; with extreme overload and high variability in the arrival pattern of patients due to absence of appointments. These findings were utilized to develop a Queuing-DEA, with output as number of doctors and consultation time as non-discretionary output since it was minimal; and length of queue and wait time of patients with inputs. Therefore, the refined DEA model will fulfil the objective of evaluating the queue continuously, as well as, reflect the exact 'required' number of doctors, at a given time.

\subsection{Data Collection Using Proposed Queuing-DEA Model}


The proposed Queuing-DEA model as shown in Section 2.3 was applied within the designated hospital in Pakistan. The data was gathered at the busy Combined Military Hospital (CMH) in Lahore, Pakistan. Data for preliminary results was collected over a period of 7 weeks $\left(23^{\text {rd }}\right.$ April to $28^{\text {th }}$ May 2014) at the Outpatients' department including GDMO clinic, Pharmacy and a few Specialist OPDs. The wait times of patients were observed from 7 am to $4 \mathrm{pm}$ on weekdays. The data consisted of a total sample of 741 patients where 37 data points were excluded due to incomplete information. To maintain patient confidentiality, the patients were referred as P1, P2 and so on. The DEA analysis has been conducted in PIM-DEA software ${ }^{1}$ (Version 3.2) and dynamic framework for implementation has been developed in Excel.

\section{Results}

Based on preliminary observations and results, the busiest Specialist OPD was observed to have excessive overloading and wait times of patients are very high; which is further compounded by less number of Specialists, with even fewer at the start of the clinic. The dataset shows that the availability of doctors varies from 2 to 5 over the day, and a total number of observations of 160 collected over different days of the week. As verified by the interviews with the Specialist OPDs administrators, the doctors need to perform some other tasks such as training sessions, ward rounds and other administrative responsibilities or paperwork. Hence, they arrive at the OPD at varying times.

The average wait time and queue length is 168 minutes and 30 respectively; with standard deviation of 41 minutes and 9 respectively. A 95\% confidence interval of wait time and queue length is (162 minutes, 174 minutes) and $(28,31)$ respectively. To further evaluate the variability in data, each wait time and queue length was individually observed, and the corresponding change in the 'required' number of doctors was noted, as shown in Figure 3; to identify the exact requirement of personnel (as shown by 'target' value in the DEA model). Results showed that two observations P065 and P140 are 100\% efficient, which showed that with 4 doctors, the wait time is between $1 \mathrm{~h}$ and $1.5 \mathrm{~h}$ and queue length is between 12 and 16, keeping the consultation time unchanged. Therefore, according to the DEA model, this combination of queuing variables represents an 'ideal' situation; which may not be adhered to all the time. But it acts as a guideline for the department administrators. A further analysis showed that with current availability at 2, P004 had a wait time of nearly $2 \mathrm{~h}$ (129 minutes) with queue length 32. Hence, the model showed that 7 doctors are required. In another case, P016 showed that the wait time increased to around $2.5 \mathrm{~h}$ (154 minutes) with a slight increase in queue length of 39 , but the requirement increased to 8 . This highlights the high variability in the wait time and length of queue, and the need to monitor them frequently, where the current DEA model proves to be very useful. For P041, the current availability was 5, but the wait time and length of queue were a high $3 \mathrm{~h}$ (171 minutes) and 43 respectively, increasing the target up to 9 . P048 showed that the wait time further increased to $3.5 \mathrm{~h}$ (223 minutes) raising the target of doctors to 12, although there was a slight decrease in queue length of 38 (see Figure 3).

\footnotetext{
${ }^{1}$ www.DEAsoftware.co.uk
} 
At this time, the queue has 'already' reached to an excessive level. Therefore, even a high availability is ineffective which will take another few hours to bring down the queue, and the model shows a further increase in target. This highlights the crucial issue of the incorrect 'timing' of bringing in more doctors. This information of required number is extremely significant for management to obtain information regarding ' $h o w$ ' many doctors are needed and 'when'. Therefore, management can determine an appropriate schedule of personnel at frequent intervals to control the queue and overload before it builds up; reducing the excessive wait times of subsequent patients.

*Insert Figures 3 here

\section{Implementation}

The current study also proposes a dynamic framework dedicated towards the practical implementation of the queuing-DEA model. This model has been developed in Microsoft Excel (Excel 2013) using Visual Basic for Applications (VBA) coding. The framework has been designed such that it evaluates the queue system each time a patient arrives, and displays the required number of doctors 'at that moment'. A screenshot of the developed model for the Specialist OPD is shown in Figure 4, and can be developed for other departments on a similar pattern. Column A requires the patient number and name, to identify a patient. The next four columns, B, C, D and E show the queuing data which needs to be entered manually by the receptionists including time in, time out, current number of doctors (this information is already available since doctors sign up at the Reception) and consultation time respectively. As soon as the patient reports at the reception, information will be entered in Column A, B and D, whereas data will be entered in $\mathrm{C}$ and $\mathrm{E}$ when patients report back before leaving the department. Additionally, queue length and wait time in Columns $\mathrm{C}$ and $\mathrm{G}$ respectively, will be calculated automatically and do not need to be entered manually.

\section{*Insert Figure 4 here}

The output-oriented DEA model has been developed in Excel for 50 patients, including the DEA mathematical programme and slack model in worksheet 1 ("DEAefficiency few values") and worksheet 2 ("DEAslack") respectively. Considering the first worksheet (see Figure 5 below), the inputs and outputs of the model are displayed in Columns D to E and F to $G$ respectively. The cell $\mathrm{N} 2$ represents the unit (patient). For the particular unit, $\mathrm{O} 3$ represents the efficiency level and Column $\mathrm{H}$ shows the weights (lambda values). N2, O3 and Column $\mathrm{H}$ are changing cells, and will display the values of the $50^{\text {th }}$ patient when the model completes its run. The formulae for the constraints (four constraints for four variables) are developed in cells J4 to M7. For instance, J4, K4, L4 and M4 represent the first constraint related to the input wait time in the Specialist clinic, and similarly, the three rows below represent other constraints. Furthermore, the lambda values will appear in Columns Q to BN, such as rows Q2 to BN2 for Patient 1 up till Q51 to BN51 for Patient 50. The efficiency levels for all 50 patients are then displayed in Column I (with percentages in Column BO). For instance, for P003, the inefficient 
unit needs to increase the output by $5 \%$ (as greater than 1) to obtain $100 \%$ efficiency, hence the efficiency level is $95 \%$.

\section{*Insert Figure 5 here}

In the second worksheet (see Figure 6), the slack model is displayed which is set up to run 'after' the DEA model in worksheet 1. Columns A to I, and cell O3 which shows the efficiency level, are copied from first to the second worksheet to be used by slack model. The objective function of the slack model is the 'sum of slacks' which is displayed in cell P8. The slack variables are set-up in cells P4, P5, P6 and P7. The constraints for slack model are set up from $\mathrm{J} 4$ to M7. When the model runs, the slacks are displayed in Columns $\mathrm{R}$ to $\mathrm{U}$ for each of the 50 patients. For instance, Patient 1 will have slacks of two inputs and outputs in cells R2, S2, T2 and U2; up till Patient 50 with values in R51, S51, T51 and U51. Using the slack and input/output values, the target values are calculated for each input and output, and displayed in Columns W to Z; and rounded off as shown in Columns AB to AE. Column AD represents the target values of the number of doctors, where this column is copied to Column $\mathrm{P}$ on worksheet 1 , to avoid switching between worksheets. The model will 're-run' every time the data of a new patient is entered, updating the required number of doctors each time.

\section{*Insert Figure 6 here}

The proposed dynamic framework is extremely beneficial for continuous monitoring of the rapidly changing queue situation due to absence of appointments, and to ensure timely action in terms of bringing in more doctors when required. This action taken earlier on, will allow for controlling the queue pre-emptively, greatly minimizing the wait times of subsequent patients; and ensuring dynamic staff allocation.

\section{Discussion}

Prior studies have evaluated walk-in patients only with respect to improving existing appointment systems. Aharonson-Daniel et al., (1996) simulated an optimal appointment system where 50 patients were allowed within every 30-minute interval, for a total of 3.5 hours; where the results showed that the wait time was over 50 minutes. Baril et al., (2016) emphasized that maximum time of 69.31 minutes was spent waiting from the total lead time of around an hour (74.76 minutes). Taleb (2019) used a lean six sigma model to suggest improvements in the appointment schedules during weekdays and weekends, reducing wait times by $67 \%$ and $63 \%$ respectively, as well as $55 \%$ reduction in walk-in patient wait time incorporated in the appointment schedule. Cayirli et al., (2019) designed access rules for an appointment schedule to decide for the number of slots to be allocated for scheduled patients after considering uncertainty due to seasonal walk-ins, leading to a reduction of wait time of 15 minutes for scheduled patients. The current study evaluated a queue system where 'all' patients are walk-in, and showed that the wait time and overload is very high. For instance, as observed at the busiest Specialist OPD, maximum patients waited for $2.5 \mathrm{~h}$ to $3.5 \mathrm{~h}$ with some 
up to nearly $4 \mathrm{~h}$, with overload of nearly 50 patients; which is nearly double the wait time of most previous works, signifying the severity of the queue situation.

The current study has utilized DEA to construct a model for queue assessment. In recent years, DEA modelling has moved away from its traditional and straightforward application in health. For instance, Wagner et al., (2003) compared the efficiency of physicians within one hospital using two DEA models. Osman et al., (2011) assessed the efficiency of nurses in an ICU, to identify best practices in order to develop suitable career development plans. Keshtkaran et al., (2014) compared the efficiency of radiology units of hospitals. These extended applications indicate the potential of DEA for assessing the efficiency of other units rather than evaluation of health institutions; however, such studies are very limited. Jafari et al., (2010) developed eight queuing models using queuing theory and used DEA to find the optimal queuing model. Safdar et al., (2016) identified the usefulness of DEA to assess a queue system in a health centre. To the best of our knowledge, these two are the only studies which have showed the utilization of DEA for a queue problem; and there are no studies so far which have shown the application of DEA in a healthcare setting to evaluate patient queue system. The current study extends DEA modelling and provides evidence of its effectiveness, specifically for a queue system of outpatients with no appointment system using real-time queuing data of patients. The preliminary observations indicated that the inadequate availability of personnel is a critical issue. Although the availability of doctors/pharmacists varies throughout the day, however, it is not synced with the excessive queuing situation. Previous DEA studies have included personnel as an input, including physicians, as well as nurses and other clinical and non-clinical professionals and nurses (Ahmed et al., 2019; Lindbauer et al., 2016; Dotoli et al., 2015). However, the current study has included number of personnel as an output, as the objective is to determine the 'required' number of personnel; given long wait times, high overload and variability in the arrival pattern of patients due to absence of appointments. It can alert the management about the 'exact' number of personnel required at a specific time to ensure reduced wait times of subsequent patients.

This study also constructs a dynamic framework for practical implementation of the proposed model. Considering previous works, some have specifically utilized Excel for improving a queue system. For instance, Wijewickrama and Takakuwa (2008) developed multiple appointment schedules in Excel using different patient sequences. Harper and Gamlin (2003) utilized Excel for preliminary analysis of patient flow systems in nine different clinics. However, when evaluating the sequencing and scheduling of routine and same-day patients Chen and Robinson (2014) recommended using Excel for post-analysis and implementation. Viana et al., (2020) utilized Excel to record service (consultation) times. The dynamic framework for current study has been developed in Excel using VBA coding such that the complex technical functions operate automatically in the background, requiring minimal effort from the users. Additionally, most users are familiar with Excel functionalities and it is currently available at the reception desks of Outpatients' department at the designated hospital. Hence, Excel is a viable option encouraging smooth and quick implementation. In a recent study, Munavalli et al., (2020) proposed a patient scheduling model with pathway optimization, where the patients will be scheduled for the respective departments/two units, when they arrive. 
In busy public hospitals of emerging economies such as Pakistan, patients arrive in a random manner and cannot be scheduled for a specific pathway beforehand, as they take different paths. This study proposes a queue management framework which uses real-time queue data, to continuously monitor the queue system in each department independently, display target number of personnel, and update automatically as new patients arrive. Utilizing this information, the management can respond to quickly changing queue situation through dynamic and efficient staff allocation. Hence, personnel can be briefly withdrawn from other tasks when queue is excessive, and when queue dies down, the management will know instantly re-allocating staff to undertake other pending activities. Consequently, the wait times of walkin patients can be minimized and overload can be controlled, in the absence of appointments.

\section{Implications for Research}

The current study addresses the crucial issue of long wait times and variable arrival pattern of patients in the absence of appointment systems; by proposing a queue assessment model which uses real-time queuing data to continuously monitor the patient flow system. The proposed model is flexible which not only evaluates the current queue situation but also proposes a solution by identifying the 'exact' number of healthcare personnel required at any given time. The current study has applied this model in a busy Specialist Outpatients' department, however, this model is generic and has the potential to be implemented in any department of hospitals with no appointment systems, which experience overcrowding and quick queue build-up as in developing economies. Furthermore, the proposed dynamic framework has been developed in a spread sheet specifically to facilitate public hospitals in emerging economies. This framework is easy to use, requires minimal effort from the users and is an effective way to record the wait times of patients. Additionally, it alerts the management about queue build-up and demonstrates the required number of healthcare personnel at a given time using real-time queue data so that the management can act immediately; such as assigning more doctors at that particular time. This will allow the management to respond instantly to sudden overcrowding, while optimally utilizing the resources (e.g. health professional). This framework, with its practical significance and user-friendly characteristics; can be easily set-up in any department of any public hospital, leading to effective health delivery.

\section{Conclusion}

This study addresses the significant issue of excessive queuing in healthcare delivery in emerging economies, where appointment systems are non-existent. Our contribution is the development of a combined queuing-DEA model that specifically targets the complex queue system of a busy public hospital of an emerging economy, Pakistan; where all patients are walk-in, with variable arrival patterns and overcrowding, and inadequate staff scheduling. This model acts as a guideline for the management as it provides the 'required' number of doctors at a given time, which would allow them to control the exponentially accumulating queue due to walk-ins earlier on; leading to reduced wait times, as well as optimized scheduling of resource personnel. The model has been applied in a Specialist OPD and its effectiveness has been demonstrated, however, this model is flexible and can be applied to any department in any hospital, where appointment systems are absent. Additionally, this study proposes a 
dynamic framework which has practical implications and allows for smooth and fast-track implementation of queuing-DEA model in any hospital, to capture the moment-to-moment change allowing for immediate action for smooth patient flow. This research extends the queuing literature by proposing a model for continuous queue monitoring in the absence of appointment system. This study extends the DEA literature by developing a dedicated DEA model for a complex queue problem and utilizing the flexible results for efficient queue management in public hospitals of developing economies. This research study can be furthered by applying this model in different hospitals, to determine the effect on managing queues with walk-in patients.

\section{References}

Aeenparast, A., Farzadi, F., Maftoon, F. and Yahyazadeh, H. (2019). Patient Flow Analysis in General Hospitals: How Clinical Disciplines Affect Outpatient Wait Times. Hospital Practices and Research, 4(4), p.128-133.

Aharonson-Daniel, L., Paul, R.J. and Hedley, A.J. (1996). Management of Queues in Out-patient Departments: The Use of Computer Simulation, Journal of Management in Medicine, 10(6), p.50-58.

Ahmed, S., Hasan, M.Z., Laokri, S., Jannat, Z., Ahmed, M.W., Dorin, F., Vargas, V. and Khan, J.A. (2019). Technical Efficiency of Public District Hospitals in Bangladesh: A Data Envelopment Analysis. Cost Effectiveness and Resource Allocation, 17(1), p.15.

Ahmed, S., Tarique, K.M. and Arif, I. (2017). Service Quality, Patient Satisfaction and Loyalty in the Bangladesh Healthcare Sector. International Journal of Health Care Quality Assurance. 30(5), p.477-488.

Anderson, K., Zheng, B., Yoon, S.W. and Khasawneh, M.T. (2015). An Analysis of Overlapping Appointment Scheduling Model in an Outpatient Clinic, Operations Research for Health Care, 4, p.5-14.

Bailey, N.T. (1952). A Study of Queues and Appointment Systems in Hospital Out-Patient Departments, with Special Reference to Waiting-Times. Journal of the Royal Statistical Society: Series B (Methodological), 14(2), p.185199.

Banker, R.D. and Morey, R.C. (1986). Efficiency Analysis for Exogenously Fixed Inputs and Outputs, Operations Research, 34(4), p.513-521.

Barghash, M. and Saleet, H. (2018). Enhancing Outpatient Appointment Scheduling System Performance when Patient NoShow Percent and Lateness Rates are High. International Journal of Health Care Quality Assurance. 31(4), p.309326.

Baril, C., Gascon, V., Miller, J. and Côté, N. (2016). Use of a Discrete-Event Simulation in a Kaizen Event: A Case Study in Healthcare, European Journal of Operational Research, 249(1), p.327-339.

Bastian, N.D., Ekin, T., Kang, H., Griffin, P.M., Fulton, L.V. and Grannan, B.C., (2017). Stochastic Multi-Objective AutoOptimization for Resource Allocation Decision-Making in Fixed-Input Health Systems. Health Care Management Science, 20(2), p.246-264.

Berenguer, G., Iyer, A.V. and Yadav, P. (2016). Disentangling the Efficiency Drivers in Country-Level Global Health Programs: An Empirical Study, Journal of Operations Management, 45, p.30-43.

Bhattacharjee, P. and Ray, P.K. (2014). Patient Flow Modelling and Performance Analysis of Healthcare Delivery Processes in Hospitals: A Review and Reflections, Computers and Industrial Engineering, 78, p.299-312.

Brahimi, M. and Worthington, D.J. (1991). Queuing Models for Outpatient Appointment Systems: A Case Study, Journal of Operational Research Society, 42(9), p.733-746.

Cayirli, T., Dursun, P. and Gunes, E.D., (2019). An Integrated Analysis of Capacity Allocation and Patient Scheduling in Presence of Seasonal Walk-Ins. Flexible Services and Manufacturing Journal, 31(2), pp.524-561.

Cayirli, T. and Veral, E. (2003). Outpatient Scheduling in Healthcare: A Review of Literature, Production and Operations Management, 12(4), p.519-549. 
Chandra, D. (2015). Reducing Waiting Time of Outdoor Patients in Hospitals Using Different Types of Models: A Systematic Survey, International Journal of Advance Research and Innovation, 3(1), p.81-87.

Charnes, A., Cooper, W.W., Lewin, A. and Seiford, L. (eds.) (1994). Data Envelopment Analysis: Theory, Methodology and Applications, Boston, MA: Kluwer Academic Publishers.

Chen, R.R. and Robinson, L.W. (2014). Sequencing and Scheduling Appointments with Potential Call-In Patients. Production and Operations Management, 23(9), p.1522-1538.

Cooper, W.W., Seiford, L.M., and Zhu, J. (2011). Data Envelopment Analysis: History, Models and Interpretations, In Handbook on Data Envelopment Analysis, $2^{\text {nd }}$ ed, USA: Springer.

Dilrukshi, P.A.D., Nirmanamali, H.D.I.M., Lanel, G.H.J. and Samarakoon, M.A.S.C. (2016). A Strategy to Reduce the Waiting Time at the Outpatient Department of the National Hospital in Sri Lanka. International Journal of Scientific and Research Publications, 6(2), p.281-287.

Dotoli M., Epicoco, N., Falagario, M. and Sciancalepore, F. (2015). A Cross-Efficiency Fuzzy Data Envelopment Analysis Technique for Performance Evaluation of Decision Making Units Under Uncertainty, Computers \& Industrial Engineering, 79, p.103-114.

Driouchi, A., (2014). Labour and Health Economics in the Mediterranean Region: Migration and Mobility of Medical Doctors. USA: IGI Global.

Feldman, J., Liu, N., Topaloglu, H., and Ziya, S. (2014). Appointment Scheduling under Patient Preference and No-show Behavior, Operations Research, 62(4), p.794-811.

Fetter, R.B. and Thompson, J.D. (1966). Patients' Waiting Time and Doctors' Idle Time in the Outpatient Setting, Health Services Research, 1(1), p. 6-90.

Golany, B. and Roll, Y. (1993). Some Extensions of Techniques to Handle Non-Discretionary Factors in Data Envelopment Analysis. Journal of productivity Analysis, 4(4), p.419-432.

Harper, P.R. and Gamlin, H.M. (2003). Reduced Outpatient Waiting Times with Improved Appointment Scheduling: A Simulation Modelling Approach, OR Spectrum, 2(2), p.207-222.

Herwartz, H. and Strumann, C. (2014). Hospital Efficiency under Prospective Reimbursement Schemes: An Empirical Assessment for the Case of Germany. The European Journal of Health Economics, 15(2), p.175-186.

Hollingsworth, B. (2012). Revolution, Evolution or Status-quo? Guidelines for Efficiency Measurement in Healthcare, Journal of Productivity Analysis, 37(1), p.1-5.

Huarng, F. and Lee, M.H. (1996). Using Simulation in Outpatient Queues: A Case Study, International Journal of Health Care Quality Assurance, 9(6), p.21-25.

Jafari, Y., Madahi, R. and Khodayifar, S. (2010). Evaluating the Performance of Multiple Comparable Queuing by Using DEA, $2^{\text {nd }}$ National Conference on Data Envelopment Analysis.

Jalal, S. and Inam-ul-Haq, (2014). Revisiting the Three Different Tiers of the Health System of Pakistan and Their Implications for the Achievement of MDGs by Pakistan, Journal of Pakistan Medical Association, 64(2), p.195200.

Javed, S.A. and Ilyas, F., (2018). Service Quality and Satisfaction in Healthcare Sector of Pakistan: The Patients' Expectations. International Journal of Healthcare Quality Assurance, 31(6), p.1-13.

Jiang, F.C., Shih, C.M., Wang, Y.M., Yang, C.T., Chiang, Y.J. and Lee, C.H., (2019). Decision Support for the Optimization of Provider Staffing for Hospital Emergency Departments with a Queue-Based Approach. Journal of Clinical Medicine, 8(12), p.2154.

Kalwar, M.A., Mari, S.I., Memon, M.S., Tanwari, A. And Siddiqui, A.A. (2020). Simulation Based Approach For Improving Outpatient Clinic Operations. Mehran University Research Journal Of Engineering \& Technology, 39(1), p.153-170.

Keshtkaran, A., Barouni, M., Ravangard, R. and Yandrani, M. (2014). Economic Efficiency of Radiology Wards Using Data Envelopment Analysis: Case Study of Iran. Health, 6(5), p.311.

Klassen, K.J. and Rohleder, T.R. (1996). Scheduling Outpatient Appointments in a Dynamic Environment, Journal of Operations Management, 14(2), p.83-101. 
Klassen, K.J. and Yoogalingam, R., (2019). Appointment Scheduling in Multi-Stage Outpatient Clinics. Health Care Management Science, 22(2), pp.229-244.

Kortbeek, N., Braaksma, A., Smeenk, F.H., Bakker, P.J. and Boucherie, R.J. (2015). Integral Resource Capacity Planning for Inpatient Care Services Based on Bed Census Predictions by Hour. Journal of the Operational Research Society, 66(7), p.1061-1076.

Kuiper, A., de Mast, J. and Mandjes, M., (2019). The Problem of Appointment Scheduling in Outpatient Clinics: A Multiple Case Study of Clinical Practice. Omega, 102122.

Lakshmi, C. and Iyer, S.A. (2013). Application of Queueing Theory in Health Care: A Literature Review. Operations Research for Health Care, 2(1-2), pp.25-39.

Lee, H. and Kim, C., (2014). Benchmarking of Service Quality with Data Envelopment Analysis. Expert Systems with Applications, 41(8), p.3761-3768.

Lindlbauer, I., Schreyögg, J. and Winter, V. (2016). Changes in Technical Efficiency after Quality Management Certification: A DEA Approach Using Difference-In-Difference Estimation with Genetic Matching in the Hospital Industry. European Journal of Operational Research, 250(3), p.1026-1036.

Liu, J.S., Lu, L.Y., Lu, W.M. and Lin, B.J. (2013). Data Envelopment Analysis 1978-2010: A Citation-Based Literature Survey. Omega, 41(1), p.3-15.

Luo, L., Zhou, Y., Han, B.T. and Li, J., (2019). An Optimization Model To Determine Appointment Scheduling Window for an Outpatient Clinic with Patient No-Shows. Health Care Management Science, 22(1), pp.68-84.

Mandelbaum, A., Momcilovic, P. and Tseytlin, Y. (2012). On Fair Routing from Emergency Departments to Hospital Wards: QED Queues with Heterogeneous Servers, Management Science, 58(7), p.1273-1291.

Marynissen, J. and Demeulemeester, E., (2019). Literature Review on Multi-Appointment Scheduling Problems in Hospitals. European Journal of Operational Research, 272(2), pp.407-419.

Mensah, J., Asamoah, D. and Tawiah, A.A. (2015). Optimizing Patient Flow and Resource Utilization in Out Patient Clinic: A Comparative Study of Nkawie Government Hospital and Aniwaa Health Center. The Journal of Applied Business and Economics, 16(3), p.181-188.

Mital, K.M. (2010). Queuing Analysis for Outpatient and Inpatient services: A Case Study, Management Decision, 48(3), p.419-439.

Mitropoulos, P., Mitropoulos, I. and Giannikos, I. (2013). Combining DEA with Location Analysis for the Effective Consolidation of Services in the Health Sector. Computers \& Operations Research, 40(9), p.2241-2250.

Munavalli, J.R., Rao, S.V., Srinivasan, A. and van Merode, G.G., (2020). Integral Patient Scheduling in Outpatient Clinics Under Demand Uncertainty to Minimize Patient Waiting Times. Health Informatics Journal, 26(1), pp.435-448.

Muñiz, M., Paradi, J., Ruggiero, J. and Yang, Z. (2006). Evaluating Alternative DEA Models Used to Control for NonDiscretionary Inputs, Computers \& operations research, 33(5), p.1173-1183.

Nappo, R.W. and Ross, E.A., (2020). Improved Outpatient Haemodialysis Adequacy Using Queued Schedules. Journal of Renal Care, 46(1), pp.62-68.

Oche, M.O. and Adamu, H. (2014). Determinants of Patient Waiting Time in the General Outpatient Department of a Tertiary Health Institution in North Western Nigeria, Annals of Medical and Health Sciences Research, 3(4), p.588-592.

Osman, I.H., Berbary, L.N., Sidani, Y., Al-Ayoubi, B. and Emrouznejad, A. (2011). Data Envelopment Analysis Model for the Appraisal and Relative Performance Evaluation of Nurses at an Intensive Care Unit, Journal of Medical System, 35(5), p.1039-1062.

Pakistan Social and Living Standards Measurement (PSLM) Survey (2018-19). Economic Survey Reveals Pakistan's Literacy Rate Increased to $60 \%$. The News (International). 11 June. Available at: https://www.thenews.com.pk/latest/671198-economic-survey-reveals-pakistans-literacy-rate-increased-to-60 [Accessed: 20 July 2020]

Peter, P.O. and Sivasamy, R. (2019). Queueing Theory Techniques and its Real Applications to Health Care SystemsOutpatient Visits. International Journal of Healthcare Management, pp.1-9. DOI: 10.1080/20479700.2019.1616890 
Perez, E., Anandhan, V. and Novoa, C., (2020). A Simulation-Based Planning Methodology for Decreasing Patient Waiting Times in Pure Walk-In Clinics. International Journal of Information Systems in the Service Sector (IJISSS), 12(3), p.34-54.

Qureshi, Z. (2020). Pakistan Surpasses Brazil to Become World's $5^{\text {th }}$ Most Populous Country. Gulf News, 12 July. Available at: https://gulfnews.com/world/asia/pakistan/pakistan-surpasses-brazil-to-become-worlds-5th-most-populouscountry-1.72557051. [Accessed: 20 July 2020]

Safdar, K.A., Emrouznejad, A. and Dey, P.K. (2016). Assessing the Queuing Process Using Data Envelopment Analysis: An Application in Health Centres. Journal of Medical Systems, 40(1), p.1-13.

Susanti, B., Syah, T.Y.R. and Sunaryanto, K. (2020). Lean Consumption Implementation for Acceleration Improvement in Executive Outpatient Wait Time over Hermina Hospital Bekasi. Journal of Multidisciplinary Academic, 4(1), p.4246.

Taleb, D.A. (2019). Implementing Lean Six Sigma in a Kuwaiti Private Hospital Yazan Al-Zain, Lawrence Al-Fandi, Mazen Arafeh, Samar Salim, Shouq Al-Quraini, Aisha Al-Yaseen. International Journal of Health Care Quality Assurance, 32(2), p.431-446.

Viana, J., Simonsen, T.B., Faraas, H.E., Schmidt, N., Dahl, F.A. and Flo, K., (2020). Capacity and Patient Flow Planning in Post-Term Pregnancy Outpatient Clinics: A Computer Simulation Modelling Study. BMC Health Services Research, 20(1), p.1-15.

Wagner, J.M., Shimshak, D.G. and Novak, M.A. (2003). Advances in Physician Profiling: The Use of DEA, SocioEconomic Planning Sciences, 37(2), p.141-163.

Wang, S., Liu, N. and Wan, G. (2020). Managing Appointment-Based Services in the Presence of Walk-In Customers. Management Science, 66(2), p. 667-686.

Wei, J., Shen, L., Yang, H.B., Qin, J.B., Huang, W., Zhang, J.J., Gong, Q.Y., Li, X.X. and Yang, T.B. (2015). Development and Validation of a Chinese Outpatient Satisfaction Questionnaire: Evidence from 46 Public General Hospitals and 5151 Outpatients. Public Health, 129(11), p.1523-1529.

Wijewickrama, A. and Takakuwa, S. (2008), Outpatient Appointment Scheduling in a Multi Facility System, In: Proceedings of the 2008 Winter Simulation Conference, ed. S. J. Mason, R. R. Hill, L. Mönch, O. Rose, T. Jefferson, J. W. Fowler, 1563-1571. Piscataway, New Jersey: IEEE.

World Bank Data (2018). Rural Population. Available at: https://data.worldbank.org/indicator/SP.RUR.TOTL.ZS. [Accessed: 20 July 2020].

World Health Statistics (2015). World Health Organisation. Available at: http://www.who.int/gho/publications/world_health_statistics/2015/en/_Accessed: 28 March 2020].

World Health Statistics (2019). World Health Organisation. Available at: https://apps.who.int/iris/bitstream/handle/10665/324835/9789241565707-eng.pdf?ua=1 [Accessed: 28 March 2020]

Yang, Z. and Paradi, J.C. (2003). Benchmarking Competitive Banking Units Using ‘Handicapped’ DEA. Omega.

Zhu, Z., Heng, B.H. and Teow, K.L. (2012). Analysis of Factors Causing Long Patient Waiting Time and Clinic Overtime in Outpatient Clinics, Journal of Medical Systems, 36(2), p.707-713. 


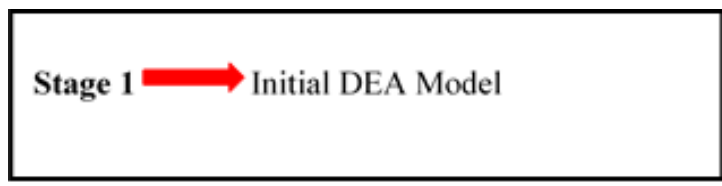

Stage $2 \longrightarrow$ Few Preliminary

Observations/Interviews and Second-stage DEA Model

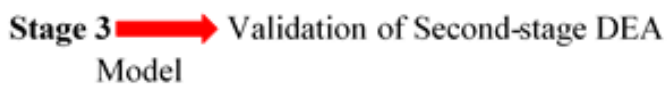

Stage $3 \longmapsto$ Validation of Second-stage DEA Model

Stage $4 \longrightarrow$ Focused Data Collection (Real-time queuing data via structured observation of Patient Flow System and interviews with staff members)

Stage $\mathbf{5} \longrightarrow$ Preliminary Findings Obtained from Focused Data Collection

Stage $6 \longrightarrow$ Refined DEA Model for Queue Assessment Obtained from Preliminary Findings

Stage $7 \longrightarrow$ Analytical Results Obtained by Applying the Proposed Model at the Three Departments at the Designated Hospital

Stage $8 \longrightarrow$ Construction of a Dynamic Framework for Practical Implementation of the Proposed Model

Figure 1: Summary of Different Stages for DEA Model Development 


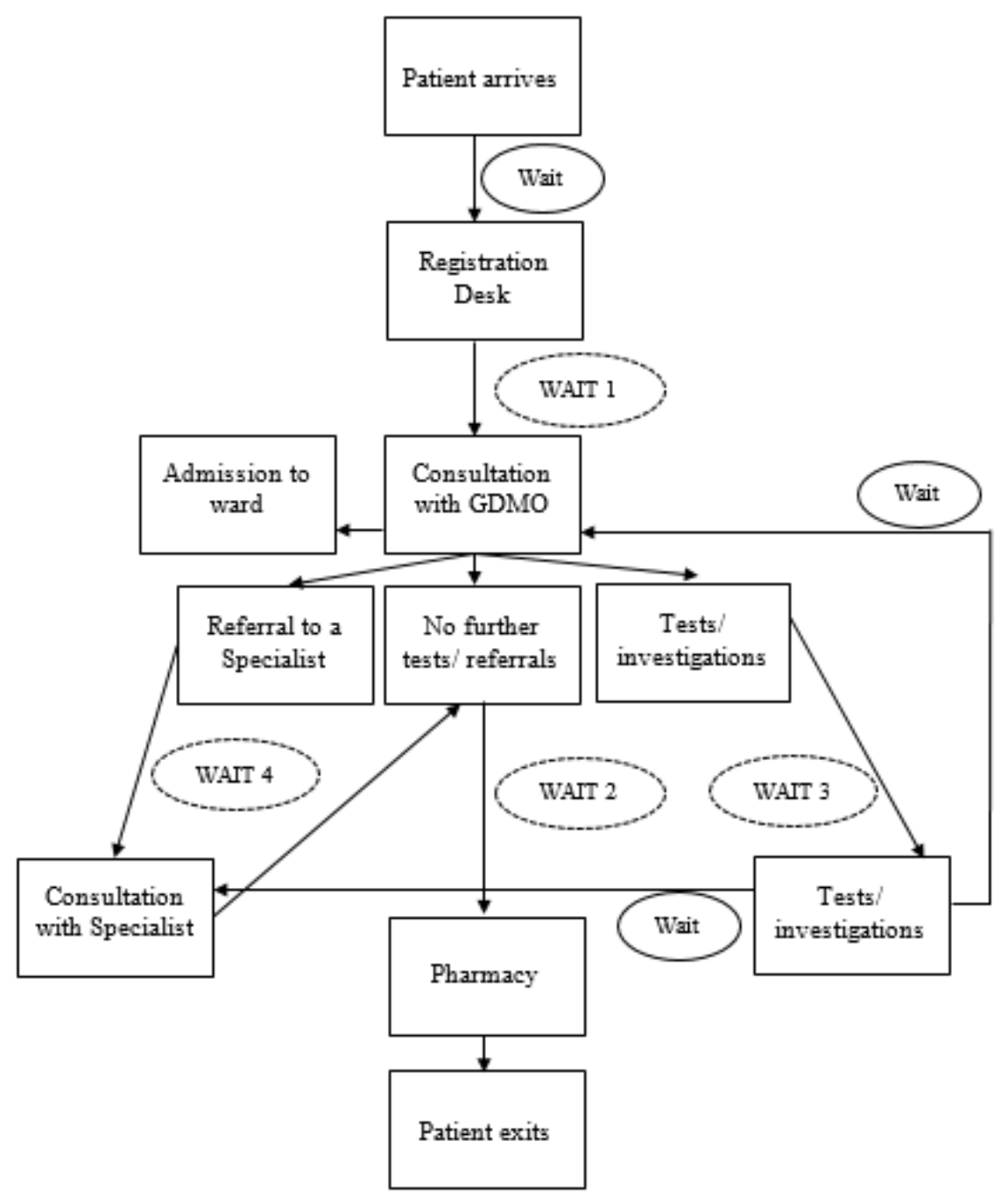

Figure 2: Patient Flow System at a Busy Public Hospital in Pakistan 


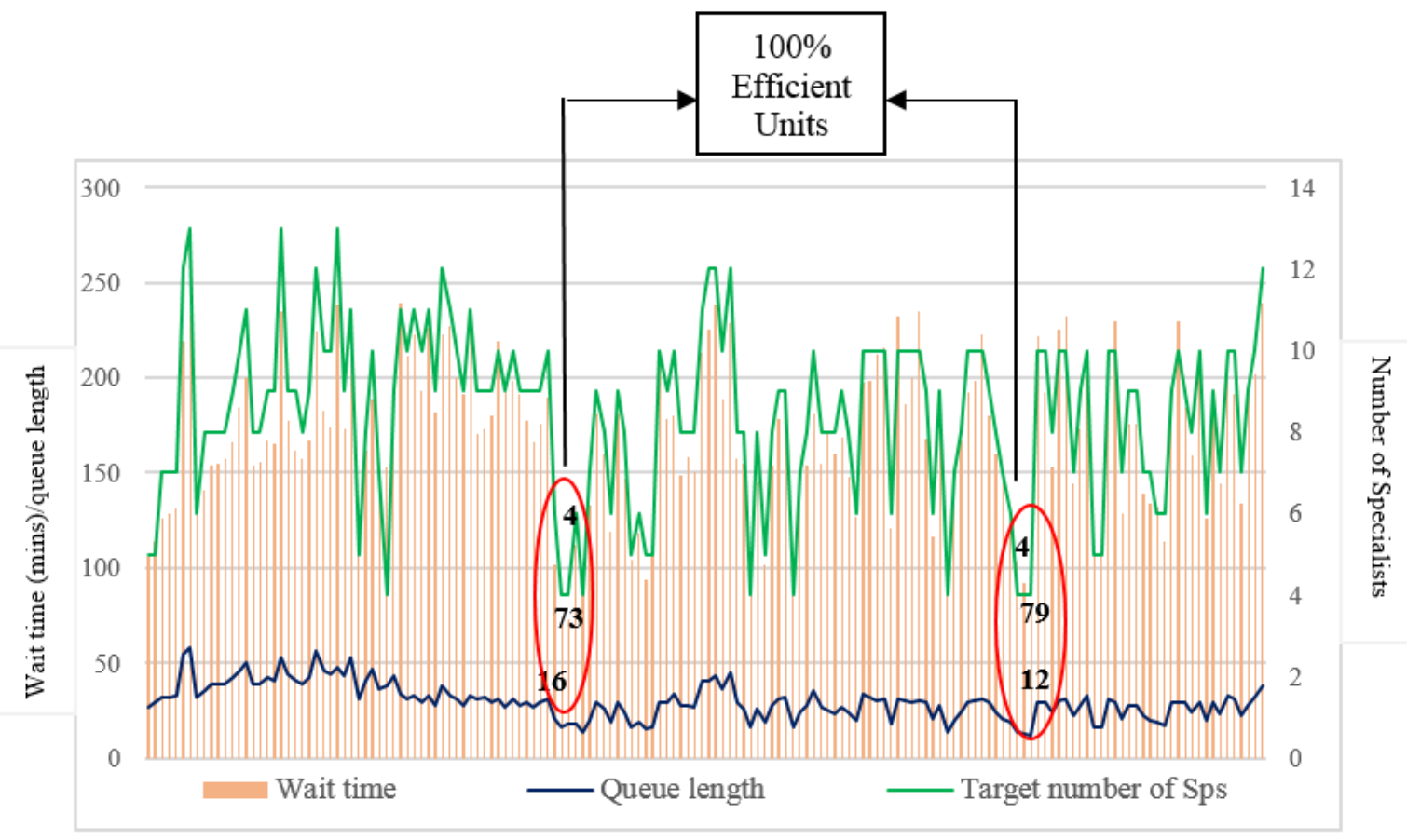

\begin{tabular}{ccc}
\hline Wait time Category & $\begin{array}{c}\text { Length of Queue } \\
\text { Category }\end{array}$ & $\begin{array}{c}\text { Required Number of } \\
\text { Specialists } \\
\text { (Specialist OPD) }\end{array}$ \\
\hline $60-90$ mins & 14 to 28 & 4 to 5 \\
\hline $90-120$ mins & 16 to 29 & 5 to 6 \\
\hline $120-150$ mins & 20 to 33 & 5 to 8 \\
\hline $150-180$ mins & 23 to 44 & 8 to 10 \\
\hline $180-210$ mins & 28 to 47 & 9 to 10 \\
\hline $210-240$ mins & 31 to 58 & 10 to 13 \\
\hline
\end{tabular}

Figure 3: Summary of Results for Busy Specialist OPD 


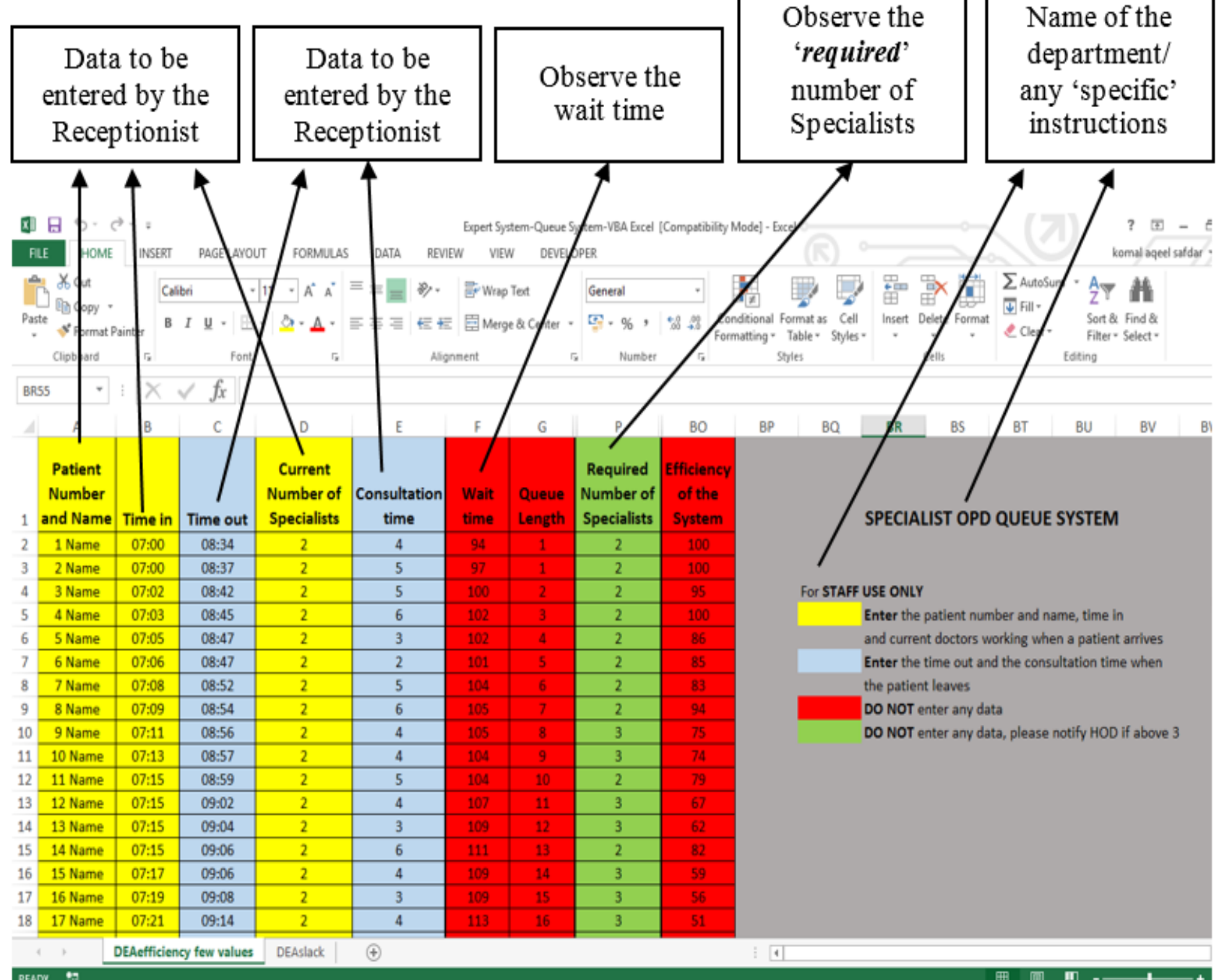

Figure 4: Proposed Dynamic Framework for Queue Assessment 


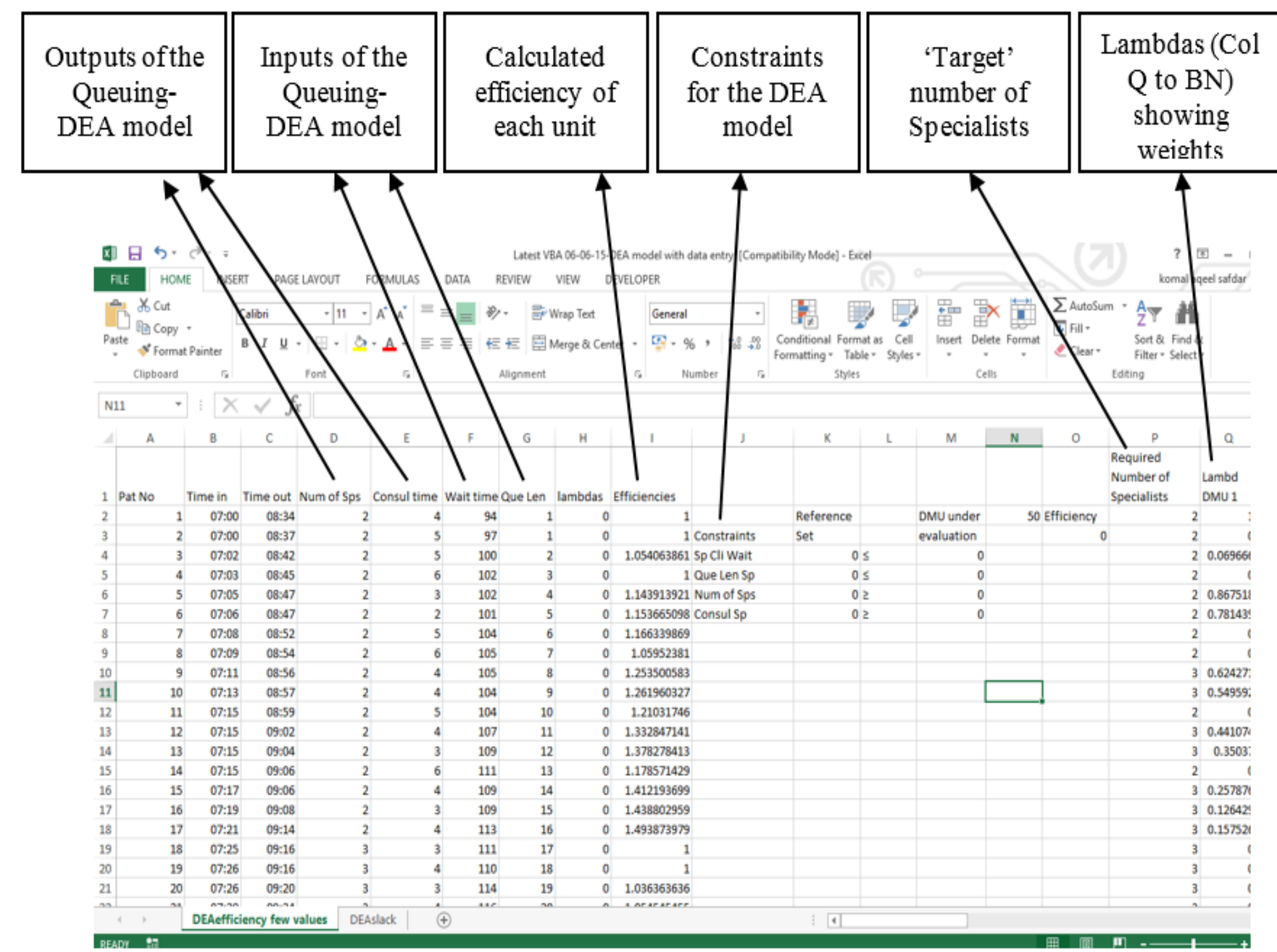

Figure 5: Model 1 of the Dynamic Framework 


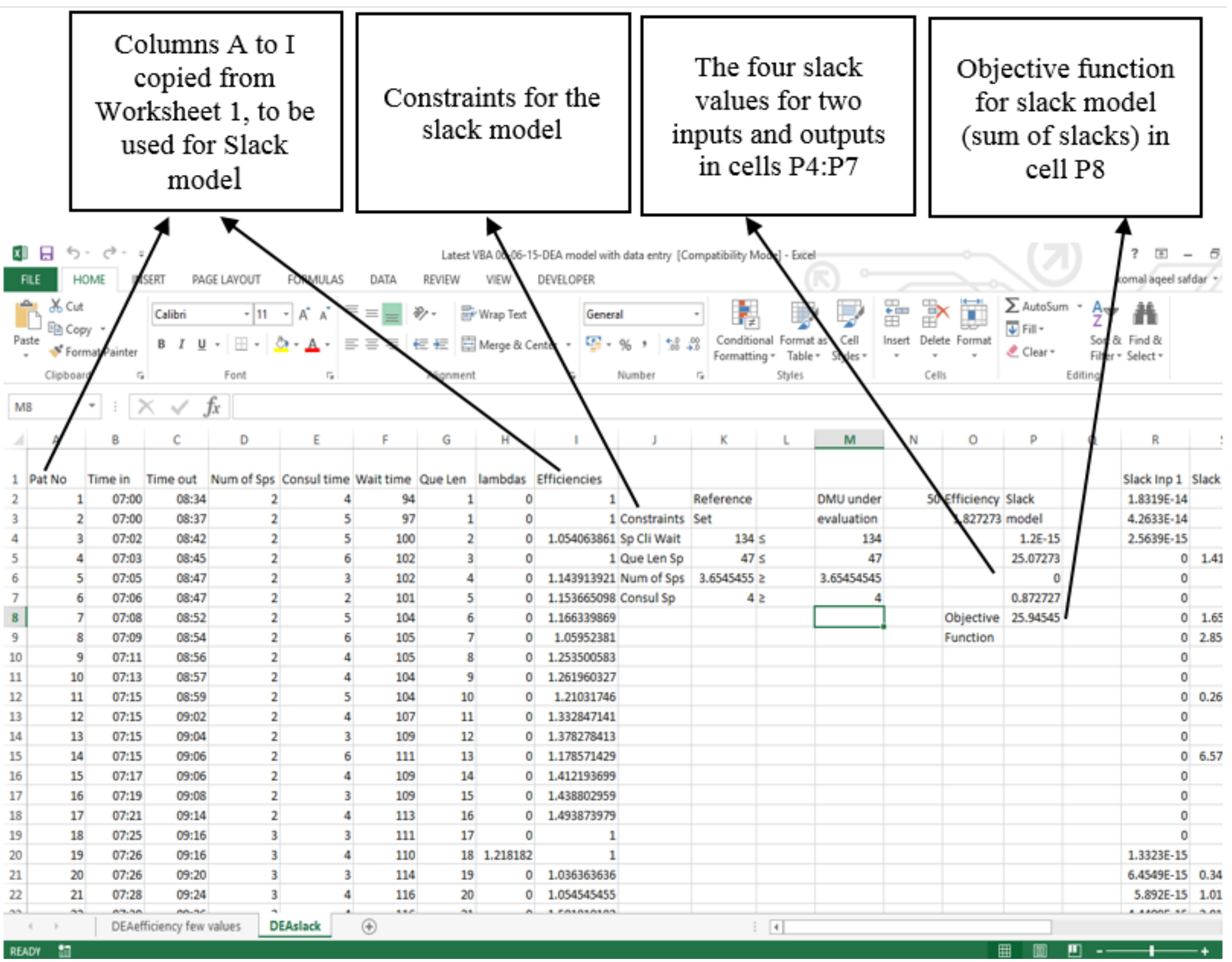

Figure 6: Model 2 of the Dynamic Framework 